\title{
MRI-Ultrasound Fusion Imaging for Diagnosis of Deep Infiltrating Endometriosis - A Critical Appraisal
}

\section{(c) (i) ()ㅜ $\ominus$}

\author{
Authors
}

Judith Berger ${ }^{1,4}$, Onno Henneman ${ }^{2}$, Johann Rhemrev ${ }^{3}$, Maddy Smeets ${ }^{3}$, Frank Willem Jansen ${ }^{4}$

\section{ABSTRACT} ity in daily practice.
Purpose It was the aim of our study to evaluate this procedure using pelvic anatomical landmarks in order to assess the accuracy of fusion imaging and to critically evaluate the applicabil-

Methods In a prospective, single center study, 10 patients with clinical signs of deep infiltrating endometriosis (DIE) were selected. We measured the distance between the landmark organ and the target shown by the software system (measurement 1). Measurement 2 depicts the distance between the landmark and the nearest calibration point. The calibration inaccuracy was measured as a third type of measurement (measurement 3).

Results Measurement 1: the average distance between the organ landmark to the target was $13.6 \mathrm{~mm}$ (range: $0-96 \mathrm{~mm}$ ). Measurement 2: in 31 of the 40 attempts (77.5\%), we could measure the distance from the landmark organ to the nearest calibration point. The average distance was $34.4 \mathrm{~mm}$ (range: 0-69 mm).

Measurement 3: A perfect match was seen in 6 of 20 attempts $(30.0 \%)$. There was a deviation in 14 of the 20 attempts $(70.0 \%)$. The mean distance was $11.1 \mathrm{~mm}$ (range: $6-23 \mathrm{~mm}$ ). Conclusion Although very promising, MRI-ultrasound fusion imaging (MUFI) currently cannot be readily implemented into daily practice as a routine evaluation of DIE.

\section{Introduction}

Endometriosis is defined as the presence of endometriotic glands outside the uterus. In addition to peritoneal endometriosis and ovarian endometriosis, a third form has been defined: deep infiltrating endometriosis (DIE), in which the endometriotic glands invade $>5 \mathrm{~mm}$ into the underlying tissue [1]. It can cause pelvic pain, dysmenorrhea, dyspareunia, dyschezia and urinary tract symptoms depending on the anatomical location [2]. An accurate diagnosis of DIE is mandatory to help the patient make a choice regarding the different treatment options: medical, surgical and/or to plan a fertility treatment [3].
MRI and ultrasound both have their advantages and disadvantages in the diagnosis of endometriosis. Transvaginal ultrasound (TVUS) is better at detecting rectal nodules (accuracy TVUS $95.8 \%$ vs. MRI 94.9\%) [4-6], whereas MRI is a better method for evaluating the extent of pelvic endometriosis with an accuracy of $90.8 \%$ [4]. Both TVUS and MRI are operator-dependent and therefore depend on the experience of the gynecologist or the radiologist for accurate staging of DIE. Combining different ultrasonic features with standard TVUS examination, such as the uterine sliding sign, hard and soft markers (for instance hydrosalpinx or loculated fluid), the mobility of the pelvic organs and tenderness-guided ultrasound 
results in more dynamic transvaginal ultrasound and may help to identify DIE easier [7-11].

Fusion imaging is a new technology, merging cross-sectional imaging data with ultrasound images in a real-time setting. MRI-ultrasound fusion imaging (MUFI) has proven useful in locating small prostate tumors: more accurate biopsies can be taken using MUFI [12-16].

Millischer et al. [17] have applied fusion imaging in the field of gynecology. Their aim was to assess the main anatomical sites of deep infiltrating endometriosis in patients with suspected active endometriosis. They showed that fusion imaging of uterosacral ligaments, rectum, posterior vaginal fornix, ureters and bladder is feasible and concluded that this technique had the potential to improve the performance of ultrasound and MRI examination. However, their conclusions were based on superimposition of both MRI and ultrasound images by visual inspection [17].

It was the aim of our study to evaluate this procedure in patients with possible abnormal anatomy by using pelvic anatomical landmarks in order to assess the accuracy of fusion imaging and critically evaluate the applicability in daily practice.

\section{Methods}

A prospective single-center study was conducted over a 6-month period. Patients with symptoms associated with endometriosis and with a strong suspicion of DIE were referred to our specialized clinic for endometriosis and were invited to participate in our study. We excluded patients with an MRI scan longer than 90 days prior to MUFI. The diagnosis of DIE was made as descried by Bazot et al. [4]: in summary: hyperintense foci on the fat-suppression T1 images with corresponding hemorrhagic foci on $\mathrm{T} 2$ images, areas of fibrosis in pelvic region, distortion of normal anatomy without any other explanation or discontinuation of normal fatty tissue between organs. The diagnostic criteria of DIE on ultrasound were in accordance with the criteria described by the IDEA consensus UOG [11]. Patients with an MRI scan with movement artifacts were excluded.

For this study, MUFI was considered to be the reference image. All MRI scans were evaluated by a single radiologist (O.H., more than 10 years of abdominal cross-sectional imaging experience). Fusion MRI-ultrasound was performed by O.H. (radiologist) and J.B. (gynecologist), both of whom are endometriosis imaging experts. The MRI exams were performed on a 1.5-T superconducting magnet (Magnetom Avantofit TM; Siemens, Erlangen, Germany) using an 18-channel RF body coil.

The MRI protocol consisted of multiplanar TSE T2-weighted images: 512 matrix, axial, sagittal and coronal with a voxel size: $0.8 \times 0.8 \times 4.0$ and axial and sagittal T1 fat-saturated breath-hold sequences: 320 matrix voxel size: $1.3 \times 1.3 \times 6.0 \mathrm{~mm}$. These protocols did not include an isovolumetric sequence. Prior to examination, patients were administrated $20 \mathrm{mg}$ butylscopolamine bromide for bowel movement inhibition. No enema was administered and patients did not fast. No contrast was used. The bladder was not emptied before MRI or MUFI. With an empty bladder, nodules in the bladder are more difficult to visualize. The filling of the bladder is comparable in both techniques.
The MRI-ultrasound fusion examination was performed with a Toshiba APLIO 500 TM system, using a vaginal convex array transducer $(5-9 \mathrm{~Hz})$ and the smart fusion TM software and hardware features. The position sensor unit was attached to the vaginal probe and a transmitter was placed near the patient during the exam. Before scanning, the axial and sagittal images were uploaded to the smart fusion TM software. To visualize both the MRI images and the transvaginal scanning images simultaneously, we used 2-point calibration on the uterus: 1 point on the fundus of the uterus, 1 point on the uterine cervix. A similar technique was used as described by Millischer et al. except they used 1-point calibration [17].

In all patients, we attempted to assess the following landmarks: Right and left ovaries (if present), posterior vaginal fornix, rectum, uterus and the urethra orifice in the bladder. We chose these landmarks because these anatomical sites are most affected by DIE, except for the urethra orifice. The latter we chose to have one site that is normally not affected by endometriosis as a 'control' landmark.

First, a mark was put on the anatomical site to be evaluated on the MRI scan (landmark organ). Next, the corresponding mark on the ultrasound image was identified (target organ). Finally, a superimposition of the two images was constructed and the distance between the target organ and the mark was measured (measurement 1). Depending on the target organ, the ultrasound probe was positioned either in the anterior (bladder, urethra) or posterior fornix (rectum, ovary, uterus, posterior fornix). Multiple superimpositions per patient were constructed.

After measurement 1 , we proceeded to measurement 2 . To evaluate the distance between the target organ and the calibration point, we visualized both the mark on the target organ and the nearest calibration point in one image. Next, the distance between the target organ and the nearest calibration point was measured (measurement 2).

Finally, during the procedure, we re-evaluated the calibration with 5-min intervals: an overlay of the two images was constructed and the distance between the calibration point on MRI and ultrasound was measured.

\section{Statistical analysis}

To evaluate the correlation between the accuracy of measurement 1 (distance between organ landmark and MRI fusion target) and measurement 2 (distance between organ landmark and calibration point), Spearman's correlation was used.

\section{Ethics}

The regional ethical committee approved our study. Written consent was obtained in all patients.

\section{Results}

We selected 20 patients with clinical suspicion of DIE who had undergone a pelvic MRI scan. 14 patients underwent an MRI scan without any movement artifacts. We invited them to participate in our study. Of those patients, 10 agreed to volunteer to come in for an extra visit and participate in our study. In 7 out of 10 patients, DIE was confirmed by histology obtained by laparoscopy. 2 patients refused laparoscopy and in 1 patient the diagnosis of DIE was rejected by MUFI. 
The mean age was 28.3 years (range: $20-36$ year). The mean time interval between MRI and fusion imaging was 42.9 days (range: 17-79 days). All measurements including calibration and recalibration were performed within 18.75 min (mean: 7:42 min, range: $4.20-18.75 \mathrm{~min}$ ) ( Table $\mathbf{1}$ ).

\section{Measurement 1}

In 37 of the 40 assessments ( $92.5 \%$ ) we could measure the distance from the landmark organ to the fusion target point. The average distance was $13.6 \mathrm{~mm}$ (range: $0-96 \mathrm{~mm}$ ). In 3 assessments, it was not possible to measure the distance, because we could not visualize the landmark organ and the target in one image.

\section{Measurement 2}

31 of the 40 assessments were complete (77.5\%), and 9 assessments were incomplete. The measurement of the distance between the landmark organ and the nearest calibration point was not feasible, since it was not possible to visualize both points in one ultrasound image. The average distance was $34.4 \mathrm{~mm}$ (range: $0-69 \mathrm{~mm})$.

\Table 1 Demographics.

\begin{tabular}{|l|l|}
\hline Age & 28.3 years (range: $20-36$ years) \\
\hline BMI & $25.6 \mathrm{~kg} / \mathrm{m} 2$ (range: $27-35.5 \mathrm{~kg} / \mathrm{m} 2)$ \\
\hline Dysmenorrhea & $9 / 10(90 \%)$ \\
\hline Dyspareunia & $6 / 10(60 \%)$ \\
\hline Dysuria & $3 / 12(30 \%)$ \\
\hline Dyschezia & $5 / 10(50 \%)$ \\
\hline $\begin{array}{l}\text { Time interval between MRI and } \\
\text { MUFI }\end{array}$ & 42.9 days (range: $17-79$ days) \\
\hline Time MUFI procedure & $7: 42$ min (range: $4.20-18.75 \mathrm{~min})$ \\
\hline
\end{tabular}
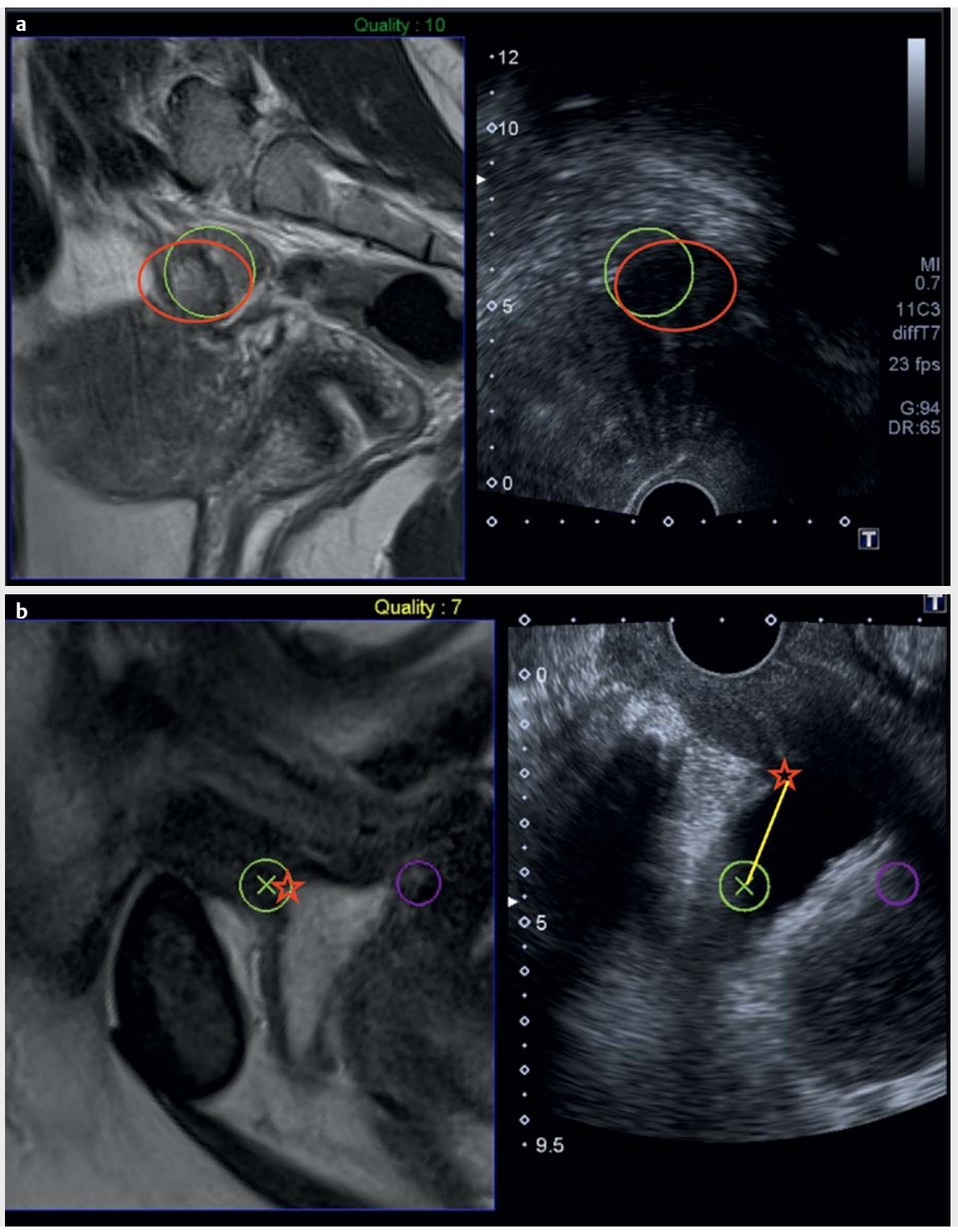

- Fig. 1 a Measurement 1: The circle was placed on the landmark organ on MRI (ovary, ellipse, left) and reappeared on the TVUS on the target organ (ovary, ellipse, right). This was a perfect match. b The small circle with X was put on the landmark organ (uretric orifice, asterix, left) and reappeared on the TVUS on another part of the bladder. The deviation was measured. The circle without $\mathrm{X}$ is the calibration point. 


\section{Measurement 3}

Every 5 min during the examinations we evaluated the calibration. We tried to match our 2-point calibration between MRI and ultrasound. In 6 of 20 attempts (30.0\%), the superimposition was perfect. There was a deviation in 14 of the 20 attempts (70.0\%). In the latter case, the mean distance was $11.1 \mathrm{~mm}$ (range: 6-23 mm).

We correlated measurement 1 (the distance between the landmark organ to the MRI fusion target shown by the software) to measurement 2 (the distance between the organ landmark and calibration point) using Spearman's correlation. Spearman's rho was $0.149(p=0.433)$.

\section{Discussion}

Our findings, based on more quantitative measurements, are comparable with the results of Millischer et al. We also found good visualization of predetermined targets[17], but the image between MRI and MUFI was not always in a perfect superimposition. We found a mean difference of $13.6 \mathrm{~mm}$ between MRI and MUFI ( Fig. 1b). The difference can be explained by multiple factors: Firstly, pelvic contents are dynamic most of the time. So, with a slightly different position of the uterus, the ovaries may have a different position too. Secondly, the TVUS probe moves organs out of their position on the MRI scan. And thirdly, patient movements may also influence measurements. To minimize the latter movement factors, we checked the calibration every 5 min. In 6 attempts we found a perfect match ( $\mathbf{F i g}$. 3a), and in 14 attempts we did not ( $\vee$ Fig. $\mathbf{3 b}$ ). In the protocol described by Millischer et al., a calibration is only performed at the beginning of the procedure[17]. In our experience, recalibration during the procedure is necessary, especially after patient movement or when the targets are further away. However, we could not establish significance, so more factors influencing the measurements are likely to be involved. To assess accuracy, a more detailed protocol utilizing bladder filling and calibration close to the target organ should be used. Also, an extra (possibly 3D) MRI sequence may contribute to the accuracy of MUFI ( Fig. 2).

Besides the need to invest in the hardware and software for this technique and in training for gynecologists using MUFI, some critical remarks need to be addressed before we implement this technique in our daily practice. Firstly and most importantly, the MRI scan needs to be free of movement artifacts and with good resolution. Secondly, for logistical reasons, the MUFI scan cannot be performed on the same day as the MRI scan, so an extra visit for the patient to the clinic needs to be planned. Finally, the cost-effectiveness of adding MUFI for the diagnosis of DIE is not well established yet. However, this was not within the scope of our study.

The question as to whether the MUFI technique makes sense for biopsies in (deep) endometriosis can be raised. For prostate cancer, MUFI has been shown to be very useful in taking biopsies. In patients with an elevated PSA, prostate cancer can be difficult to visualize on ultrasound, while endorectal MRI is superior. Since taking a biopsy during MRI scanning is not possible, MUFI is an excellent technique to take more accurate biopsies than using ultrasound alone $[12,14-16]$. Similar results have been obtained in the diagnosis of hepatocellular carcinoma [18]. However, routine biopsies are not common practice in patients with DIE. Therefore, this advantage of MUFI is questionable for this reason. However, when a discrepancy between TVUS and MRI is found, MUFI may have a place in the diagnostic pathway for DIE.

As mentioned earlier, the advantage of using more dynamic transvaginal ultrasound is the ability to evaluate the 'sliding sign', mobility of the pelvic organs and site-specific pain [6-10]. This will provide the gynecologist additional information which contributes to the assessment of the correct stage of disease. Moreover, an accurate diagnosis of DIE will help the patient make an informed decision regarding the therapeutic options [3]. Since TVUS for the assessment of DIE has a learning curve [19], not every hospital has a well-trained ultrasonographer for detecting DIE. One of the biggest challenges for gynecologists is the lack of anatomic orien-

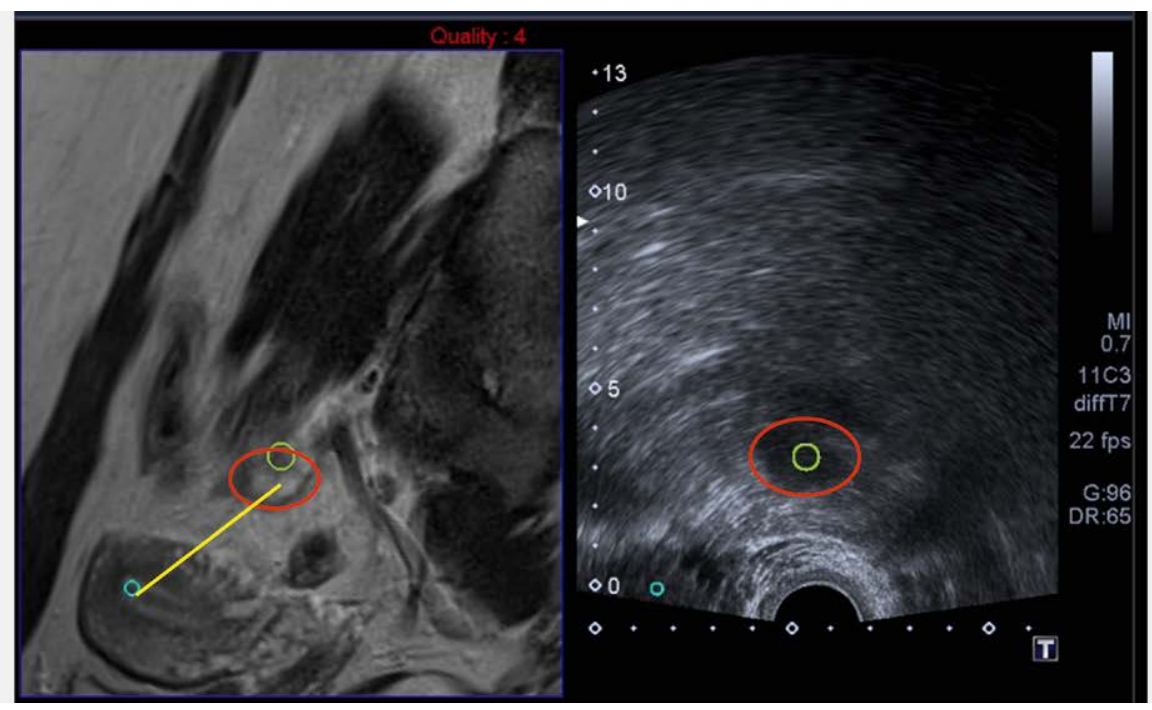

- Fig. 2 Measurement 2: The distance between the landmark organ (ovary, ellipse, left) and the nearest calibration point (uterus, small circle) was measured. 

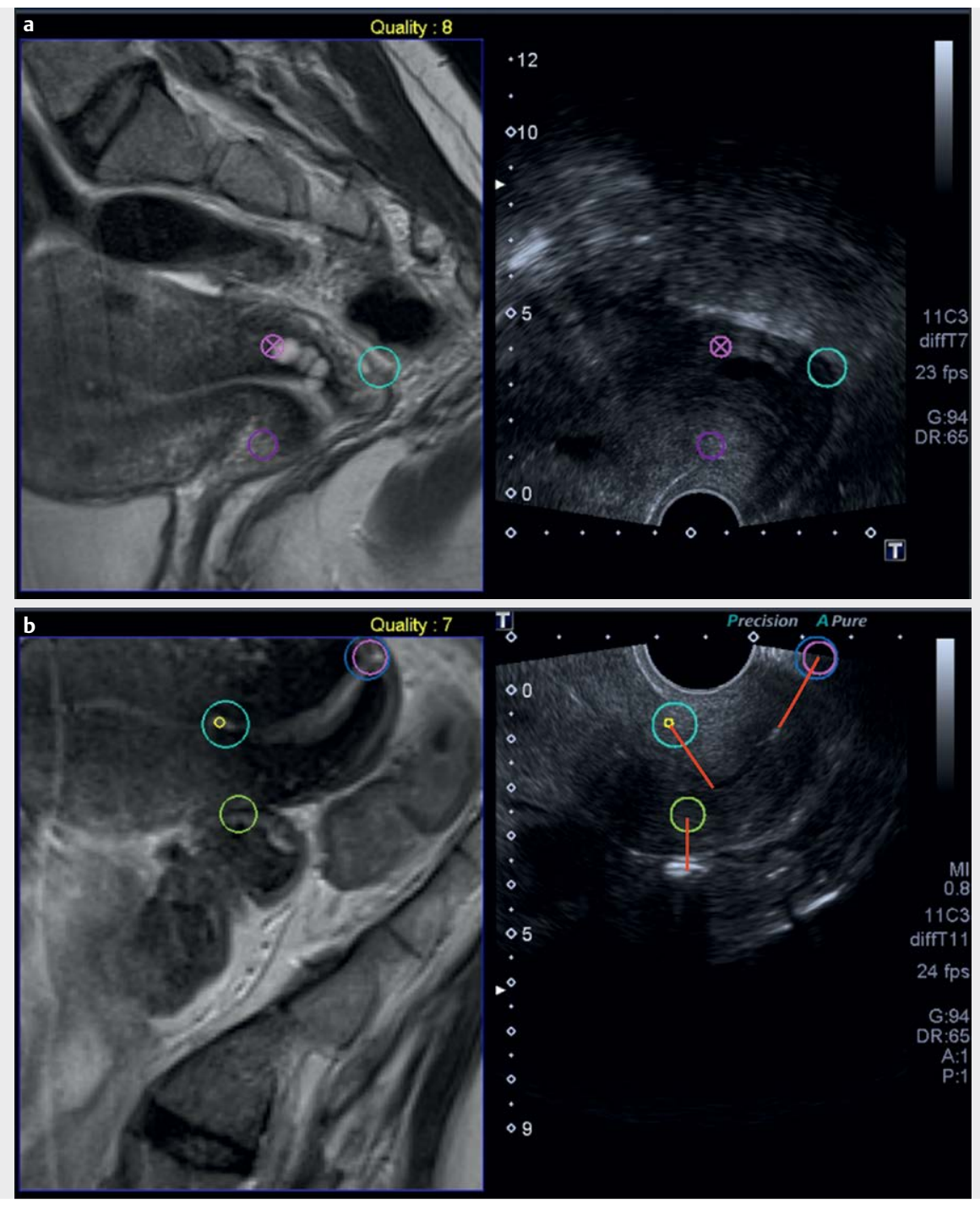

Fig. 3 a Measurement 3: Calibration check with 2-point calibration. Circle with X (ovula Nabothi, cervix) big circle: posterior fornix. The right part of the picture (i. e., the TVUS image) has been zoomed in to focus on the landmarks. b Calibration check with 2 calibration points: circle with small circle inside: internal cervical ostium, circle with big circle inside: external cervical ostium, solitary circle: landmark organ (bowel)

tation when interpreting MR imaging for endometriosis. The fieldof-view is turned by 90 degrees compared to TVUS imaging. In clinics where a dedicated sonographer is not present, MUFI may contribute to the diagnosis and staging of DIE by linking the images of MRI and TVUS. However, in our opinion, there is no place for the routine use of MUFI in the diagnosis of endometriosis.

To conclude, fusion imaging for DIE is a feasible, reproducible new technique. These initial results show promising accuracy and combine the positive effects of both MRI and TVUS imaging. However, before implementing this technique in daily practice, improvement in the software and more precise clinical conditions need to be met. Recently, Yavariabdi et al. [20] reported on the mapping and characterizing endometrial implants by registering 2D TVUS to 3D pelvic MRI, using advanced computerized modelling approaches. Although very promising, at present MUFI cannot be readily implemented into daily practice as a routine evaluation of DIE. The cost-effectiveness of adding MUFI for the diagnosis of
DIE can be questioned. However, this was not within the scope of this article.

\section{Conflict of Interest}

The authors declare no conflict of interest.

\section{References}

[1] Johnson NP, Hummelshoj L, Adamson GD, Keckstien J, Taylor HS, Abrao MS, Bush D, Kiesel L, Tamimi R, Scharpe-Timms KL, Rombauts L, Giudice LC. World endometriosis society consensus on the classification of endometriosis. Hum Reprod 2017; 2: 15-324

[2] Chapron D, Fauconnier A, Vierira M, Barakat H, Dousset B, Pansini V, Vacher-Lavenu MC, Dubuisson JB. Anatomical distribution of deeply infiltrating endometriosis: Surgical implications and proposition for a classification. Human Reprod 2003; 18: 157-161 
[3] Holland TK, Cutner A, Saridogan Mavrelos D, Pateman K, Jurkovic D. Ultrasound mapping of pelvic endometriosis: Does the location and number of lesions affect the diagnostic accuracy? A multicentre diagnostic accuracy study. BMC Womens health 2013; 13: 43

[4] Bazot M, Daraï E. Sonography and MR imaging for the assessment of deep pelvic endometriosis. J Minim Invasive Gynecol 2005; 12: 178-185

[5] Hudelist G, English ], Thomas AE, Tinelli A, Singer CF, Keckstein J. Diagnostic accuracy of transvaginal ultrasound for non-invasive diagnosis of bowel endometriosis: Systematic review and meta-analysis. Ultrasound Obstet Gynecol 2011; 3: 257-263

[6] Guerriero S, Ajossa S, Orozco R, Perniciano M, Jurado M, Melis GB, Alcazar JL. Accuracy of transvaginal ultrasound for diagnosis of deep endometriosis in the rectosigmoid: Systematic review and metaanalysis. Ultrasound Obstet Gynecol 2016; 47: 281-289

[7] Guerriero S, Ajossa S, Gerada M, Virgilio B, Angioni S, Melis GB. Diagnostic value of transvaginal 'tenderness-guided' ultrasonography for the prediction of location of deep endometriosis. Human Reprod 2008; 23: 2452-2457

[8] Hudelist G, Fritzer N, Staettner S, Tammaa A, Tinelli A, Sparic R, Keckstein J. Uterine sliding sign: A simple sonographic predictor for presence of deep infiltrating endometriosis of the rectum. Ultrasound Obstet Gynecol 2013; 41: 692-695

[9] Okaro E, Condous G, Khalid A, Timmerman D, Amye L, Huffel SV, Bourne T. The use of ultrasound-based 'soft markers' for the prediction of pelvic pathology in women with chronic pelvic pain - can we reduce the need for laparoscopy? BJOG 2006; 113: 251-256

[10] Reid S, Lu C, Casikar I, Reid G, Abbott ], Cario G, Chou D, Kowalski D, Cooper M, Condous G. Prediction of pouch of Douglas obliteration in women with suspected endometriosis using a new real-time dynamic transvaginal ultrasound technique: The sliding sign. Ultrasound Obstet Gynecol 2013; 41: 685-691

[11] Guerriero S, Condous G, van den Bosch T, Valentin L, Leione FP, Van Schoubroeck D, Exacoustos C, Installé AJ, Martins WP, Abraoi MS, Hudelist G, Bazot M, Alcazar JL, Conçalves MO, Pascual MA, Ajossa S, Savelli L, Dunham R, Reid S, Menakaya U, Bourne T, Ferrero S, Leon M, Bignardi T, Holland T, Jurkovic D, Benacerraf B, Osuga Y, Somigliana E, Timmerman D. Systematic approach to sonographic evaluation of the pelvis in women with suspected endometriosis, including terms, definitions and measurements: A consensus opinion from the International Deep Endometriosis Analysis (IDEA) group. Ultrasound Obstet Gynecol 2016; 48: 318-332
[12] Kaplan I, Oldenburg NE, Meskell P, Blake M, Church P, Holupka EJ. Real time MRI-ultrasound image guided stereotactic prostate biopsy. Magn Reson Imaging 2002; 3: 295-299

[13] Sonn GA, Margolis DJ, Marks LS. Target detection: Magnetic resonance imaging-ultrasound fusion-guided prostate biopsy. Urol Oncol 2014; 6: 903-911

[14] Wu J, Ji A, Xie B, Wang X, Zhu Y, Wang J, Yu Y, Zheng X, Liu B, Xie L. Is magnetic resonance/ultrasound fusion prostate biopsy better than systematic prostate biopsy? An updated meta- and trial sequential analysis. Oncotarget 2015; 41: 43571-43580

[15] Gayet M, van der Aa A, Beerlage HP, Schrier BPh, Mulders PFA, Wijkstra $\mathrm{H}$. The value of magnetic resonance imaging and ultrasonography (MRI/US)-fusion biopsy platforms in prostate cancer detection: A systematic review. BJU Int 2015; 3: 392-400

[16] Mendhiratta N, Rosenkrantz AB, Meng X, Wysock JS, Fenstermaker M, Huang R, Deng FM, Melamed J, Zhou M, Huang WC, Lepor H, Taneja SS. Magnetic resonance imaging-ultrasound fusion targeted prostate biopsy in a consecutive cohort of men with no previous biopsy: Reduction of over detection through improved risk stratification. J Urol 2015; 6: 1601-1606

[17] Millischer AE, Salomon LJ, Santulli P, Borghese B, Sousset B, Chapron C. Fusion imaging for evaluation of deep infiltrating endometriosis: Feasibility and preliminary results. Ultrasound Obstet Gynecol 2015; 46: $109-117$

[18] Park HJ, Lee MW, Lee MH, Hwang J, Kang TW, Lim S, Rhim H, Lim HK. Fusion imaging-guided percutaneous biopsy of focal hepatic lesions with poor conspicuity on conventional sonography. J ultrasound med 2013; 32: 1557-1563

[19] Piessens S, Healey M, Maher P, Tsaltas J, Rombouts L. Can anyone screen for deep infiltrating endometriosis with transvaginal ultrasound? Aust N Z Obstet Gynaecol 2014; 5: 462-468

[20] Yavariabdi A, Bartoli A, Samir C, Artigues M, Canis M. Mapping and characterizing endometrial implants by registering $2 \mathrm{D}$ transvaginal ultrasound to 3D pelvic magnetic resonance images. Comput Med Imaging Graph 2015; 45: 11-12 